\title{
Structure of the $\mathrm{X}(3872)$ and its Isospin Symmetry Breaking
}

\author{
M. Takizawa ${ }^{1, \mathrm{a}}$ and S. Takeuchi ${ }^{2}$ \\ 1 Showa Pharmaceutical University, Machida, Tokyo 194-8543, Japan \\ 2 Japan College of Social Work, Kiyose, Tokyo 204-8555, Japan
}

\begin{abstract}
In order to understand the structure of the $X(3872)$, we have studied the effects of the $c \bar{c}$ charmonium core state coupling to the $D^{0} \overline{D^{0 *}}$ and $D^{+} D^{-*}$ molecule states. We determine the coupling strength so as to reproduce the observed mass of the the $X(3872)$. The isospin symmetry breaking is introduced by the mass differences of the neutral and charged $D$ mesons. The obtained structure of the $X(3872)$ is about $15 \%$ of $c \bar{c}$ charmoniumu, $72 \%$ of the isoscalar $D \overline{D^{*}}$ molecule and $13 \%$ of the isovector $D \overline{D^{*}}$ molecule which explains observed properties of the $X(3872)$ well.
\end{abstract}

\section{Introduction}

The $X(3872)$ state was first observed in 2003 by Belle [1] and confirmed by CDFII [2], D0 [3] and BaBar [4] collaborations. Since then, it has received much attention because the $X(3872)$ seems to be difficult to explain its structure by a simple $c \bar{c}$ bound state using the quark potential model and is considered as the candidate of the exotic state [5]. Many structures of the $X(3872)$ have been suggested such as a tetraquark structure and a $D^{0} \bar{D}^{0 *}$ molecule.

Another improtant property of the $X(3872)$ is its isospin structure. Belle have measured the branching fractions [6]

$$
\frac{B r\left(X \rightarrow \pi^{+} \pi^{-} \pi^{0} J / \Psi\right)}{B r\left(X \rightarrow \pi^{+} \pi^{-} J / \Psi\right)}=1.0 \pm 0.4 \pm 0.3
$$

It suggests the strong isospin violation. Here two pion mode is from the isovector $\rho$ meson origin and three pi mode is from the isoscalar $\omega$ meson origin. M. Suzuki has estimated the kinematical suppression factor and obtained the production amplitude ratio [7]

$$
\left|\frac{A(\rho J / \Psi)}{A(\omega J / \Psi)}\right|=0.27 \pm 0.02
$$

Usual size of the isospin symmetry breaking is at most a few \%. Thus, we should study the origin of this strong isospin symmetry breaking.

One of the authers (S. T.) has studied the $X(3872)$ using a quark potential model by introducing an extra $(q \bar{q})$ pair to a $c \bar{c}$ system [8] and found a bound state of $q \bar{q} c \bar{c}$ with $J^{P C}=1^{++}$. The purpose of the present work is to make the situation of the $X(3872)$ clear by studying the effects of the $c \bar{c}$ core state coupling to $D^{0} \overline{D^{0 *}}$ and $D^{+} D^{-*}$ molecule states. This approach complements the quark model approach.

\footnotetext{
a e-mail: takizawa@ac.shoyaku.ac.jp
}

\section{Wavefunction of $X(3872)$}

We consider the $X(3872)$ state is a mixture of the $c \bar{c}$ core state, the $D^{0} \overline{D^{0 *}}$ hadronic molecule satate and the $D^{+} D^{-*}$ hadronic molecule states. The observed mass of the $X(3872)$ is $(3872.2 \pm 0.8) \mathrm{MeV}$ which is about $0.4 \mathrm{MeV}$ above the $D^{0} \overline{D^{0 *}}$ threshold $(3871.81 \pm 0.36) \mathrm{MeV}$. In order to calculate the wavefunction of the $X(3872)$, we assume that the mass of the $X(3872)$ is about $1 \mathrm{MeV}$ below the $D^{0} \overline{D^{0 *}}$ threshold.

The wavefunction of the $X(3872)$ in the center of the mass frame is represented by

$$
|X\rangle=c_{1}|c \bar{c}\rangle+c_{2}\left|D^{0} \overline{D^{0 *}}\right\rangle+c_{3}\left|D^{+} D^{-*}\right\rangle .
$$

The $D^{0} \overline{D^{0 *}}$ and $D^{+} D^{-*}$ molecule states are given by

$$
\begin{aligned}
\left|D^{0} \overline{D^{0 *}}\right\rangle & =\int d^{3} \mathbf{q} \varphi_{0}(\mathbf{q})\left|D^{0} \overline{D^{0 *}}(\mathbf{q})\right\rangle, \\
\left|D^{+} D^{-*}\right\rangle & =\int d^{3} \mathbf{q} \varphi_{+}(\mathbf{q})\left|D^{+} D^{-*}(\mathbf{q})\right\rangle,
\end{aligned}
$$

where the normalization of the states are

$$
\begin{aligned}
\left\langle D^{0} \overline{D^{0 *}}\left(\mathbf{q}^{\prime}\right) \mid D^{0} \overline{D^{0 *}}(\mathbf{q})\right\rangle & =\left\langle D^{+} D^{-*}\left(\mathbf{q}^{\prime}\right) \mid D^{+} D^{-*}(\mathbf{q})\right\rangle \\
& =\delta^{3}\left(\mathbf{q}^{\prime}-\mathbf{q}\right) .
\end{aligned}
$$

Here $\varphi_{0}(\mathbf{q})$ and $\varphi_{+}(\mathbf{q})$ are the momentum represation of the wavefunctions of the $D^{0} \overline{D^{0 *}}$ and $D^{+} D^{-*}$ hadronic molecule states respectively. The charge conjugation + states are asuumed throughout this paper.

We introduce the monopole form factor type couplings between the $c \bar{c}$ core state and the $D \overline{D^{*}}$ states in the isospin symmetric manner.

$$
\left\langle D^{0} \overline{D^{0 *}}(\mathbf{q})|V| c \bar{c}\right\rangle=\left\langle D^{+} D^{-*}(\mathbf{q})|V| c \bar{c}\right\rangle=\frac{g}{\mathbf{q}^{2}+\Lambda^{2}} .
$$


We ignore other interactions for simplicity. The schrödinger equation we have solved is

$$
\begin{gathered}
\left(\begin{array}{ccc}
m_{c \bar{c}}-E & V & V \\
V & m_{D^{0}}+m_{D^{0 *}}+\frac{\hat{p}^{2}}{2 \mu_{0}}-E & 0 \\
V & 0 & m_{D^{+}}+m_{D^{-*}}+\frac{\hat{p}^{2}}{2 \mu_{+}}-E
\end{array}\right) \\
\quad \times\left(\begin{array}{c}
c_{1}|c \bar{c}\rangle \\
c_{2} \mid D^{0} \overline{\left.D^{0 *}\right\rangle} \\
c_{3}\left|D^{+} D^{-*}\right\rangle
\end{array}\right)=\left(\begin{array}{l}
0 \\
0 \\
0
\end{array}\right),
\end{gathered}
$$

with

$$
\frac{1}{\mu_{0}}=\frac{1}{m_{D^{0}}}+\frac{1}{m_{D^{0 *}}}, \quad \frac{1}{\mu_{+}}=\frac{1}{m_{D^{+}}}+\frac{1}{m_{D^{-*}}} .
$$

As for the $c \bar{c}$ core state, we consider it is the $J^{P C}=1^{++}$ state and its mass is $3950 \mathrm{MeV}$ from the Godfy and Isgur's result of the quark potential model calculation for the $2{ }^{3} \mathrm{P}_{1}$ $c \bar{c}$ state [9].

Let us discuss our numerical results. The parameters of the model are the cutoff $\Lambda$ and the coupling constant $g$. We take $\Lambda=0.3 \mathrm{GeV}$ and $0.5 \mathrm{GeV}$ which are the typical hadron sizes. The coupling constant $g$ is determined so as to reproduce the observed mass of the $X(3872)$, namely, $3871 \mathrm{MeV}$. For the case of $\Lambda=0.3(0.5) \mathrm{GeV}$, we obtained $g=0.0094(0.0185) \mathrm{GeV}^{3 / 2}$. In the case of the softer cutoff, the coupling is weaker.

The wavefunction we have obtained for the cutoff $\Lambda=$ $0.3 \mathrm{GeV}$ is

$$
\begin{aligned}
|X\rangle & =0.327|c \bar{c}\rangle+0.882\left|D^{0} \overline{D^{0 *}}\right\rangle+0.339\left|D^{+} D^{-*}\right\rangle \\
& =0.327|c \bar{c}\rangle+0.863\left|D \overline{D^{*}} ; I=0\right\rangle+0.384\left|D \overline{D^{*}} ; I=1\right\rangle,
\end{aligned}
$$

and for the cutoff $\Lambda=0.5 \mathrm{GeV}$ is

$$
\begin{aligned}
|X\rangle & =0.382|c \bar{c}\rangle+0.858\left|D^{0} \overline{D^{0 *}}\right\rangle+0.344\left|D^{+} D^{-*}\right\rangle \\
& =0.382|c \bar{c}\rangle+0.850\left|D \overline{D^{*}} ; I=0\right\rangle+0.363\left|D \overline{D^{*}} ; I=1\right\rangle .
\end{aligned}
$$

The cutoff dependence of the admixture of the different componets is small. We obtain the large isospin symmetry breaking and is consistent with the experimental observation Eq. (2).

The calculated coordinate space represation of the wavefunctions of the $D^{0} \overline{D^{0 *}}$ and $D^{+} D^{-*}$ hadronic molecule states $r \varphi(r)_{0}$ and $r \varphi(r)_{+}$are shown in Fig. 1. Fig. 1 shows that the $D^{0} \overline{D^{0 *}}$ bound state is very widely spread object and one can safely call it "hadronic molecule". The size of the $D^{+} D^{-*}$ bound state is smaller than that of the $D^{0}$ $\overline{D^{0 *}}$ bound state. However it is much bigger than the usual charmonium.

\section{Spectrum}

In this section, we consider the transiton strength $S(E)$ of the weak decay of $B$ meson; $B \rightarrow X(3872) K$ and we normalize the $S(E)$ to the production of the $2^{3} P_{1} c \bar{c}$ state by

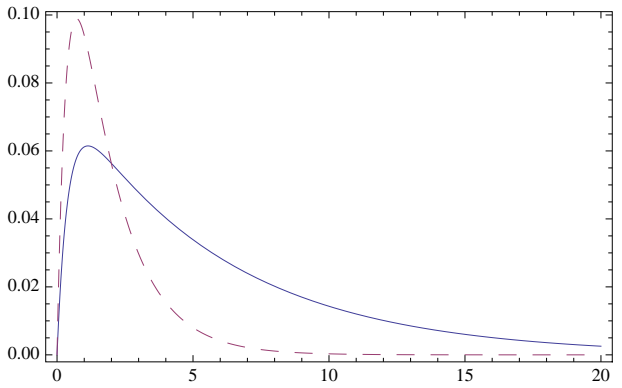

Fig. 1. The solid line shows $r \varphi(r)_{0}$ and the dashed line shows $r \varphi(r)_{+}$. The horizontal axis is in units of fm.

the weak decay. Then, the $S(E)$ is expressed as follows.

$$
S(E)=\frac{-1}{\pi} \operatorname{Im}\langle c \bar{c}|G(E)| c \bar{c}\rangle,
$$

with the Green's function;

$$
G(E)=\frac{1}{E-\hat{H}-i \varepsilon} .
$$

The state $|c \bar{c}\rangle$ represents the center of mass system of the $2^{3} P_{1} c \bar{c}$ state with the normalization $\langle c \bar{c} \mid c \bar{c}\rangle=1$. Using the free Green's functions and the interaction given in Eq. (7), the Green's function is represented as follows.

$$
\begin{gathered}
G(E)=G_{1}^{0}+G_{1}^{0} V G_{2}^{0} V G_{1}^{0}+G_{1}^{0} V G_{3}^{0} V G_{1}^{0}+\cdots, \\
G_{1}^{0}(E)=\frac{1}{E-m_{c \bar{c}}-i \varepsilon}, \\
G_{2}^{0}(E)=\frac{1}{E-m_{D^{0}}-m_{D^{0 *}}-\frac{\hat{p}^{2}}{2 \mu_{0}}-i \varepsilon}, \\
G_{3}^{0}(E)=\frac{1}{E-m_{D^{+}}-m_{D^{-*}}-\frac{\hat{p}^{2}}{2 \mu_{+}}-i \varepsilon} .
\end{gathered}
$$

The interection $V$ gives rise to the attractive force between $D$ and $\overline{D^{*}}$ effectively and the bound state exists. If the attraction is weak and the bound state is very shallow, then, such state is just the observed $\mathrm{X}(3872)$. As mentioned in Sect. 2, we have fixed the strength of the interaction $V$ so as to reproduce the observed $X(3872)$ mass.

On the other hand, the $2^{3} P_{1} c \bar{c}$ core state becomes resonance. Since such resonance is not observed experimentally, the width of this resonance should be large enough.

The calculated transition strength for the cutoff $\Lambda=0.3$ $\mathrm{GeV}$ is shown in Fig. 2. The $c \bar{c}$ core state of the bare mass of $3950 \mathrm{MeV}$ becomes the resonance state and its peak position is about $25 \mathrm{MeV}$ shifted up.

We show the transition strength in the case of the cutoff $\Lambda=0.5 \mathrm{GeV}$ in Fig. 3. One can find the difference of the shape of the resonance from that for the $\Lambda=0.3 \mathrm{GeV}$ case. The mass region of $3950 \mathrm{MeV}$, the resonance of $J^{P C}=1^{++}$ has not been observed. Therefore the cutoff $\Lambda=0.5 \mathrm{GeV}$ case is more favorable. In the case of the harder cutoff, one needs stronger coupling between the $c \bar{c}$ core state and $D \overline{D^{*}}$ molecule state so as to reproduce the $X(3872)$ mass. Because of this strong coupling, the resonance state around $3950 \mathrm{MeV}$ disappeared. 
$19^{\text {th }}$ International IUPAP Conference on Few-Body Problems in Physics

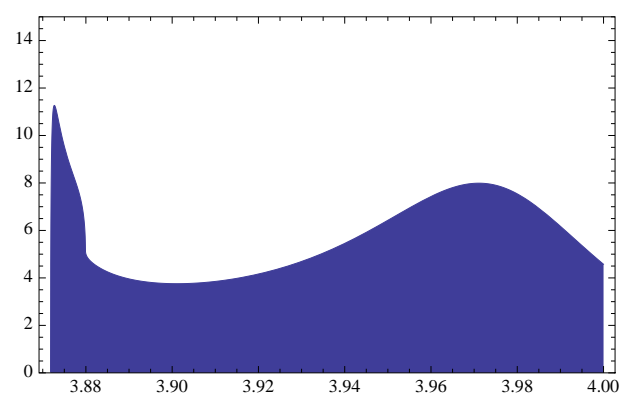

Fig. 2. The calculated transition strength $S(E)$ for the cutoff $\Lambda=0.3 \mathrm{GeV}$. The horizontal axis is in units of GeV. The $S(E)$ is shown above the $D^{0} \overline{D^{0 *}}$ threshold. The bound state below the $D^{0}$ $\overline{D^{0 *}}$ threshold, i.e., $X(3872)$ is not shown in figure.

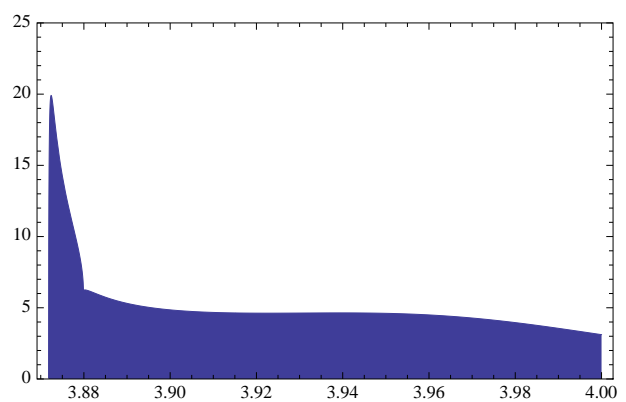

Fig. 3. The calculated transition strength $S(E)$ for the cutoff $\Lambda=0.5 \mathrm{GeV}$. The horizontal axis is in units of $\mathrm{GeV}$. The $S(E)$ is shown above the $D^{0} \overline{D^{0 *}}$ threshold. The bound state below the $D^{0}$ $\overline{D^{0 *}}$ threshold, i.e., $X(3872)$ is not shown in figure.

\section{Discussions and Summary}

We have studied the structure of the $X(3872)$. We have introduced the interactions between the $c \bar{c}$ core state and the $D \overline{D^{*}}$ molecule state. This interaction effectively gives rise to the attractive force between $D$ and $\overline{D^{*}}$ and it forms the bound state of the $D$ and $\overline{D^{*}}$. Since the $c \bar{c}$ core state cannot couple to the charged $D \overline{D^{*}}$ states, no charged partners of the $X(3872)$ exist.

The effect of the isospin symmetry breaking is introduced by the mass difference between the neutral and charged $D$ and $D^{*}$ mesons. It gives rise to the large isospin mixing in the structure of the $X(3872)$ state and our result is consistent with the experimental observation.

In the wavefunction of the $X(3872)$ we have obtained, there is about $15 \%$ of the $c \bar{c}$ core component. It helps up to explain the production rate of the $X(3872)$. If the $X(3872)$ is purely the $D \overline{D^{*}}$ molecule state, the observed production rate of the $X(3872)$ seems to be too large.

Concerning the quark potential model predictions of the charmonium states, the predicted masses are meaningful. However, if the predicted state is above the open charm threshold, one should consider the decay width of that state when you compare the theoretical results with experimental observations.

In order to confirm the present picture of the $X(3872)$, the analyses of the multi-body decay of the $X(3872)$ is im- portant since it gives us the information of the size of the $X(3872)$.

\section{References}

1. S. -K. Choi, et al., [Belle Collaboration], Phys. Rev. Lett. 91, (2003) 262001

2. D. E. Acosta, et al., [CDF II Collaboration], Phys. Rev. Lett. 93, (2004) 072001

3. V. M. Abazov, et al., [D0 Collaboration], Phys. Rev. Lett. 93, (2004) 162002

4. B. Aubert, et al., [BABAR Collaboration], Phys. Rev. D71, (2005) 071103

5. For a review, see E. S. Swanson, Phys. Rep. 429, (2006) 243

6. K. Abe, et al., [Belle Collaboration], arXiv:hepex/050537

7. M. Suzuki, Phys. Rev. D72, (2005) 114013

8. S. Takeuchi, Prog. Theor. Phys. Suppl. 168, (2007) 107

9. S. Godfrey and N. Isgur, Phys. Rev. D32, (1985) 189 